\section{Why quadratic log-log dependence is ubiquitous and what next}

\section{Quadratic $\log -\log$ dependence}

\author{
Sean R. Aguilar and Vladik Kreinovich \\ Department of Computer Science,
} University of Texas at El Paso, El Paso, Texas, USA, and

\author{
Uyen Pham \\ Faculty of Mathematical Economics, University of Economics and Law, \\ Vietnam National University, Ho ChiMinh City, Vietnam
}

\begin{abstract}
Purpose - In many real-life situations ranging from financial to volcanic data, growth is described either by a power law - which is linear in log-log scale or by a quadratic dependence in the log-log scale. The purpose of this paper is to explain this empirical fact.

Design/methodology/approach - The authors use natural scale invariance requirements.

Findings - In this paper, the authors used natural scale invariance requirement to explain the ubiquity of quadratic log-log dependencies. The authors also explain what to do if quadratic log-log models turn out to be insufficiently accurate. In this case, scale-invariance requirements lead to dependencies which in the log-log scale take cubic, 4 th order, etc. form.
\end{abstract}

Originality/value - To the best of authors' knowledge, this is the first theoretical explanation of the empirical quadratic log-log dependence.

Keywords Financial growth, Quadratic log-log dependence, Scale invariance, Volcanic activity

Paper type Research paper

\section{Formulation of the problem}

Predictions: a typical situation. One of the main objective of science and engineering is to predict the future state of the world - i.e. the future values of the quantities that describe this state - and to come up with measures that lead to the most favorable future state. For example, we want to predict tomorrow's weather - and if it will be catastrophic in a given area, we need to plan corresponding closings and, if needed, evacuations. We want to predict next year's gross domestic product (GDP) - and if the current trends predict a crisis, we want to come up with measures that would prevent this crisis - or at least decrease its severity.

(C) Sean R. Aguilar, Vladik Kreinovich and Uyen Pham. Published in Asian Journal of Economics and Banking. Published by Emerald Publishing Limited. This article is published under the Creative Commons Attribution (CC BY 4.0) licence. Anyone may reproduce, distribute, translate and create derivative works of this article (for both commercial and non-commercial purposes), subject to full attribution to the original publication and authors. The full terms of this licence may be seen at http:// creativecommons.org/licences/by/4.0/legalcode

This work was supported in part by the National Science Foundation grants 1623190 (A Model of Change for Preparing a New Generation for Professional Practice in Computer Science) and HRD1242122 (Cyber-ShARE Center of Excellence). 
AJEB

5,1

In some situations, we can predict the future values of some quantities with high accuracy. For example, we can predict solar eclipses centuries ahead.

However, such situations are rare. In most real-life situations, we cannot make exact predictions: e.g. when we predict the weather, there are many factors that affect tomorrow's weather, and that we, at present, do not know. In such situations, we can predict, at best, the probabilities of future values of the corresponding quantity. These probabilities can be described, e.g. by the probability density function (pdf) $\rho(x)$. Such situations are typical in economics and finance; they are also typical in geosciences - e.g. in predicting volcanic activity, they are typical in many other application areas.

Stationary vs non-stationary situations. In some cases - e.g. in celestial mechanics or, for a reasonably short period, in weather prediction - the corresponding probabilities remain the same day after day and year after year. So, the probability density function $\rho_{t}(x)$ remains the same for all moments of time $t: \rho_{t}(x)=\rho(x)$.

However, in many other cases, the situation changes with time: on average, the values $x$ grow with time. This happens with economic characteristics such as GDP or stock prices; this happens with volcanic activity when the volcano becomes more and more active. In many such cases, the shape of the probability distribution remains the same, but the scale changes. In other words, at each moment of time $t$, the distribution of $x$ is similar to the initial $(t=1)$ distribution of the quantity $\frac{x}{C(t)}$ for some increasing function $C(t)$, i.e. $\rho_{t}(x)$ should be proportional to $\rho_{1}\left(\frac{x}{C(t)}\right)$. The coefficient of proportionality can be easily found from the condition that the overall probability should be equal to 1 , i.e. that $\int \rho_{t}(x) d x=1$. Thus, we get:

$$
\rho_{t}(x)=\frac{1}{C(t)} \cdot \rho\left(\frac{x}{C(t)}\right) .
$$

How $C(t)$ depends on time: empirical fact. In many practical situations, the growth $C(t)$ is described by the power law:

$$
C(t)=A \cdot t^{b},
$$

for some constants $A$ and $b$. If we take the logarithm of both sides of this formula, we can conclude that in the log-log scale, the power law becomes a linear dependence:

$$
\ln (C(t))=b \cdot \ln (t)+\ln (A) .
$$

In other cases, we have a more complex dependence:

$$
C(t)=A \cdot t^{b(t)}
$$

where:

$$
b(t)=b_{0}+b_{1} \cdot \ln (t) .
$$

In this case, in log-log-scale, we have:

$$
\ln (C(t))=b(t) \cdot \ln (t)+\ln (A)=b_{0} \cdot \ln (t)+b_{1} \cdot(\ln (t))^{2}+\ln (A) .
$$


In other words, we have a quadratic log-log dependence, of which the linear log-log dependence (3) is a particular case corresponding to $b_{1}=0$.

Such dependencies are really ubiquitous: e.g. an empirical analysis provided in Mariani et al. (2020) has shown that many real-life dependencies, ranging from economic to volcanic data, follow these formulas. This naturally leads to the following questions:

Natural questions:

Q1. How can we explain the ubiquity of the dependence (4)-(5)?

Q2. When the match with this formula is not perfect, what more accurate formula should we try?

In this paper, we provide answers to both questions.

\section{Analysis of the problem: why power law?}

Why power law. In many cases, the dependence of $C(t)$ on $t$ is described by the power law. So, before we analyze the more general question of why the general dependence (4)-(5) is ubiquitous, let us analyze why power law is frequently observed.

Scale invariance: idea. We are interested in learning how the growth function $C(t)$ depends on time $t$. In our analysis, we use numerical values of $C(t)$ and numerical values of time $t$. Numerical values of time depend on the measuring unit: we get different values if we use years, quarters, months, days, etc. If we replace the original unit with the one that is $\lambda$ times smaller, then all numerical values of time $t$ are replaced with new values $\lambda \cdot t$. For example, if we replace years with months, then in the new units, the period of two years becomes $12 * 2=24$ months.

In many cases, we do not have a preferred unit for measuring time. In such situations, it is reasonable to require that the dependence between $C$ and $t$ remains the same if we change the unit for measuring time. This requirement is known as scale invariance.

The requirement of scale invariance is typical in physics: e.g. the formula $d=v \cdot t$ describing the relation between distance $d$, velocity $v$ and time $t$ remains true, no matter what units we select for measuring time, e.g. hours or seconds.

Of course, we cannot simply assume that the value $C(t)$ remains the same: the growth in two years is clearly different from the growth in two months. This can be easily explained on the example of the above physics formula: if we change the unit for time, then, for the formula to remain valid, we need to also appropriately change the units for other quantities: e.g. replace the velocity unit from $\mathrm{km} / \mathrm{hour}$ to $\mathrm{km} / \mathrm{sec}$. In our cases, this means that if we change a unit for time, then the formula $C=C(t)$ should remain valid if we also appropriately change the unit for $C$, into a new unit which is $\mu$ times smaller, for some $\mu$ depending on $\lambda$.

In other words, if we have:

$$
C=C(t)
$$

then, for every $\lambda$, we should also have:

$$
C^{\prime}=C\left(t^{\prime}\right)
$$

where:

$$
C^{\prime}=\mu(\lambda) \cdot C \text { and } t^{\prime}=\lambda \cdot t
$$



equation (8), we conclude that $\mu(\lambda) \cdot C=C(\lambda \cdot t)$. Substituting equation (7) for $C$ into this formula, we conclude that:

$$
C(\lambda \cdot t)=\mu(\lambda) \cdot C(t) .
$$

In real life, the dependencies are usually continuous, and for continuous functions, it is known that all solutions of the functional equation $(10)$ have the form $C(t)=A \cdot t^{b}$; (Aczél and Dhombres, 2008).

Thus, we indeed have a natural explanation for the power law.

\section{How to explain quadratic log-log dependence}

Main idea. As we have mentioned earlier, the main reason why the real-life processes are probabilistic is that the actual value $x(\mathrm{t})$ depends also on some characteristics that we do not know. In particular, this means that the value $C(\mathrm{t})$ also depends on such characteristics $y_{1}, \ldots, y_{n}: C=C\left(t, y_{1}, \ldots, y_{n}\right)$.

Let us first consider the simplest such case; when we consider only the dependence of one such characteristics $y_{1}$, then $C=C\left(t, y_{1}\right)$.

Let us apply scale invariance to this situation. Now, in addition to time $t$, we have another quantity $y_{1}$ for which we can also select different measuring units. It is, therefore, reasonable to require that no matter how we change both unit for $t$ and unit for $y_{1}$, we will get the same dependence.

In precise terms, for every $\lambda>0$ and $\lambda_{1}>0$, there exists a value $\mu\left(\lambda, \lambda_{1}\right)$ such that if:

$$
C=C\left(t, y_{1}\right),
$$

then:

$$
C^{\prime}=C\left(t^{\prime}, y_{1}^{\prime}\right)
$$

where:

$$
t^{\prime}=\lambda \cdot t, y_{1}^{\prime}=\lambda \cdot y_{1}, C^{\prime}=\mu\left(\lambda, \lambda_{1}\right) \cdot C .
$$

Let us analyze this situation. Substituting equation (13) into equation (12), we conclude that:

$$
C\left(\lambda \cdot t, \lambda_{1} \cdot y_{1}\right)=\mu\left(\lambda, \lambda_{1}\right) \cdot C .
$$

Substituting equation (11) for $C$ into equation (14), we get:

$$
C\left(\lambda \cdot t, \lambda_{1} \cdot y_{1}\right)=\mu\left(\lambda, \lambda_{1}\right) \cdot C\left(t, y_{1}\right) .
$$

For each $y_{1}$, by taking $\lambda_{1}=1$, we conclude that:

$$
C\left(\lambda \cdot t, y_{1}\right)=\mu(\lambda, 1) \cdot C\left(t, y_{1}\right) .
$$

Thus, for each $y_{1}$, the function $C_{y_{1}}(t) \stackrel{\text { def }}{=} C\left(t, y_{1}\right)$ satisfies equation (10). Thus, based on the result cited in the previous section, we have: 


$$
C_{y_{1}}(t)=C\left(t, y_{1}\right)=A\left(y_{1}\right) \cdot t^{b\left(y_{1}\right)},
$$

for some $A$ and $b$ depending on $y_{1}$. In particular, in log-log scale, we get:

\section{Quadratic} $\log -\log$ dependence

$$
\ln \left(C\left(t, y_{1}\right)\right)=b\left(y_{1}\right) \cdot \ln (t)+\ln \left(A\left(y_{1}\right)\right) .
$$

Similarly, for every $t$, we can take $\lambda=1$ and get:

$$
C\left(t, \lambda_{1} \cdot y\right)=\mu\left(1, \lambda_{1}\right) \cdot C\left(t, y_{1}\right) .
$$

Thus, for each $t$, the function $C_{t}\left(y_{1}\right) \stackrel{\text { def }}{=} C\left(t, y_{1}\right)$ satisfies equation (10). Thus, based on the result cited in the previous section, we have:

$$
C_{t}\left(y_{1}\right)=C\left(t, y_{1}\right)=A^{\prime}(t) \cdot y_{1}^{b^{\prime}(t)},
$$

for some $A^{\prime}$ and $b^{\prime}$ depending on $t$. In particular, in log-log scale, we get:

$$
\ln \left(C\left(t, y_{1}\right)\right)=b^{\prime}(t) \cdot \ln \left(y_{1}\right)+\ln \left(A^{\prime}(t)\right) .
$$

Equation (18) and (21) describe the same expression $\ln \left(C\left(t, y_{1}\right)\right)$. By equating these expressions for two different values $t_{1}<t_{2}$, we conclude that:

$$
\begin{aligned}
& b\left(y_{1}\right) \cdot \ln \left(t_{1}\right)+\ln \left(A\left(y_{1}\right)\right)=b^{\prime}\left(t_{1}\right) \cdot \ln \left(y_{1}\right)+\ln \left(A^{\prime}\left(t_{1}\right)\right) \\
& b\left(y_{1}\right) \cdot \ln \left(t_{2}\right)+\ln \left(A\left(y_{1}\right)\right)=b^{\prime}\left(t_{2}\right) \cdot \ln \left(y_{1}\right)+\ln \left(A^{\prime}\left(t_{2}\right)\right) .
\end{aligned}
$$

Subtracting equation (22) from equation (23), we get:

$$
b\left(y_{1}\right) \cdot\left(\ln \left(t_{2}\right)-\ln \left(t_{1}\right)\right)=\left(b^{\prime}\left(t_{2}\right)-b^{\prime}\left(t_{1}\right)\right) \cdot \ln \left(y_{1}\right)+\left(\ln \left(A^{\prime}\left(t_{2}\right)\right)-\ln \left(A^{\prime}\left(t_{1}\right)\right) .\right.
$$

So, by dividing both sides by the difference $\ln \left(t_{2}\right)-\ln \left(t_{1}\right)$, we get:

$$
b\left(y_{1}\right)=c_{1} \cdot \ln \left(y_{1}\right)+c_{2},
$$

where we denoted:

$$
c_{1}=\frac{b^{\prime}\left(t_{2}\right)-b^{\prime}\left(t_{1}\right)}{\ln \left(t_{2}\right)-\ln \left(t_{1}\right)} \text { and } c_{2}=\frac{\ln \left(A^{\prime}\left(t_{2}\right)\right)-\ln \left(A^{\prime}\left(t_{1}\right)\right)}{\ln \left(t_{2}\right)-\ln \left(t_{1}\right)} .
$$

Similarly, if we multiply equation (23) by $\ln \left(t_{1}\right)$, equation (22) by $\ln \left(t_{2}\right)$ and subtract the results, we get:

$$
\begin{aligned}
\ln \left(A\left(y_{1}\right)\right) \cdot\left(\ln \left(t_{2}\right)-\ln \left(t_{1}\right)\right)= & \left(b^{\prime}\left(t_{2}\right) \cdot \ln \left(t_{1}\right)-b^{\prime}\left(t_{1}\right) \cdot \ln \left(t_{2}\right)\right) \cdot \ln \left(y_{1}\right) \\
& +\left(\ln \left(A^{\prime}\left(t_{2}\right)\right) \cdot \ln \left(t_{1}\right)-\ln \left(A^{\prime}\left(t_{1}\right)\right) \cdot \ln \left(t_{2}\right)\right),
\end{aligned}
$$


AJEB $\quad$ Hence:
5,1

$$
\ln \left(A\left(y_{1}\right)\right)=c_{3} \cdot \ln \left(y_{1}\right)+c_{4},
$$

where we denoted:

$$
c_{3}=\frac{b^{\prime}\left(t_{2}\right) \cdot \ln \left(t_{1}\right)-b^{\prime}\left(t_{1}\right) \cdot \ln \left(t_{2}\right)}{\ln \left(t_{2}\right)-\ln \left(t_{1}\right)}
$$

and:

$$
c_{4}=\frac{\ln \left(A^{\prime}\left(t_{2}\right)\right) \cdot \ln \left(t_{1}\right)-\ln \left(A^{\prime}\left(t_{1}\right)\right) \cdot \ln \left(t_{2}\right)}{\ln \left(t_{2}\right)-\ln \left(t_{1}\right)} .
$$

Substituting equation (25) and (28) into equation (18), we conclude that:

$$
\begin{aligned}
& \ln \left(C\left(t, y_{1}\right)\right)=\left(c_{1} \cdot \ln \left(y_{1}\right)+c_{2}\right) \cdot \ln (t)+\left(c_{3} \cdot \ln \left(y_{1}\right)+c_{4}\right) \\
& =c_{4}+c_{2} \cdot \ln (t)+c_{3} \cdot \ln \left(y_{1}\right)+c_{1} \cdot \ln (t) \cdot \ln \left(y_{1}\right) .
\end{aligned}
$$

If we now assume that the dependence of $y_{1}$ on $t$ is also scale invariant, then the result of the previous section shows that:

$$
y_{1}=A^{\prime \prime} \cdot t^{b^{\prime \prime}}
$$

for some $A^{\prime \prime}$ and $b^{\prime \prime}$, i.e. in $\log -\log$ form:

$$
\ln \left(y_{1}\right)=b^{\prime \prime} \cdot \ln (t)+\ln \left(A^{\prime \prime}\right) .
$$

Substituting equation (31) into equation (30), we get:

$$
\ln (C(t))=\ln \left(C\left(t, y_{1}(t)\right)\right)=C_{0}+C_{1} \cdot \ln (t)+C_{2} \cdot(\ln (t))^{2},
$$

where:

$$
C_{0}=c_{4}+c_{3} \cdot \ln \left(A^{\prime \prime}\right), C_{1}=c_{2}+c_{3} \cdot b^{\prime \prime}+c_{1} \cdot \ln \left(A^{\prime \prime}\right), C_{2}=c_{1} \cdot b^{\prime \prime} .
$$

Thus, we indeed explained the quadratic log-log dependence.

\section{What next?}

Let us use scale invariance. In general, we have a dependence:

$$
C=C\left(t, y_{1}, \ldots, y_{n}\right),
$$

on $n \geq 1$ auxiliary quantities. In this general case, scale invariance means for every $\lambda>0$ and for all possible values $\lambda_{1}>0, \ldots, \lambda_{n}$, there exists a value $\mu\left(\lambda, \lambda_{1}, \ldots, \lambda_{n}\right)$ such that if equation (34) is satisfied, then:

$$
C^{\prime}=C\left(t^{\prime}, y_{1}^{\prime}, \ldots, y_{n}^{\prime}\right),
$$


where:

\section{Quadratic $\log -\log$}

$$
t^{\prime}=\lambda \cdot t, y_{1}^{\prime}=\lambda \cdot y_{1}, \ldots, y_{n}^{\prime}=\lambda \cdot y_{n}, C^{\prime}=\mu\left(\lambda, \lambda_{1}, \ldots, \lambda_{n}\right) \cdot C .
$$

What we can derive from scale invariance. Similar to the previous section, we can, thus, conclude that the expression $\ln \left(C\left(t, y_{1}, \ldots, y_{n}\right)\right)$ is linear in $\ln (t)$, linear in $\ln \left(y_{1}\right), \ldots$, and linear in $\ln \left(y_{n}\right)$. Thus, it is a multi-linear function:

$$
\begin{aligned}
& \ln \left(C\left(t, y_{1}, \ldots, y_{n}\right)\right)=c_{0}+c_{t} \cdot \ln (t)+\sum_{i=1}^{n} c_{i} \cdot \ln \left(y_{i}\right)+ \\
& \sum_{i=1}^{n} c_{t, i} \cdot \ln (t) \cdot \ln \left(y_{i}\right)+\sum_{i<j} c_{i, j} \cdot \ln \left(y_{i}\right) \cdot \ln \left(y_{j}\right)+\ldots+ \\
& c_{t, 1, \ldots, n} \cdot \ln (t) \cdot \ln \left(y_{1}\right) \cdot \ldots \cdot \ln \left(y_{n}\right) .
\end{aligned}
$$

If we assume that the dependence of each auxiliary quantity $y_{i}$ on $t$ is also scale invariant, then we get:

$$
\ln \left(y_{i}\right)=b_{i}^{\prime \prime} \cdot \ln (t)+\ln \left(A_{i}^{\prime \prime}\right)
$$

for some values $b_{i}^{\prime \prime}$ and $A_{i}^{\prime \prime}$. Substituting equation (38) into equation (37), we conclude that:

$$
\ln (C(t))=C_{0}+C_{1} \cdot \ln (t)+C_{2} \cdot(\ln (t))^{2}+\ldots+C_{n+1} \cdot(\ln (t))^{n+1} .
$$

Resulting recommendation. So, if quadratic log-log dependence (corresponding to $n=1$ ) is too inaccurate, we need to try cubic log-log dependence (corresponding to $n=2$ ), then, if needed, fourth-order $\log$-log dependence corresponding to $n=3$, etc.

\section{References}

Aczél, J. and Dhombres, J. (2008), Functional Equations in Several Variables, Cambridge University Press.

Mariani, M.C., Asante, P.K., Bhuyian, M.A.M., Beccar-Varela, M.P., Jaroszewicz, S. and Tweneboah, O. K. (2020), "Long-range correlations and characterization of financial and volcanic time series", Mathematics, Vol. 8 No. 3, pp. 441.

\section{Corresponding author}

Vladik Kreinovich can be contacted at: vladik@utep.edu

For instructions on how to order reprints of this article, please visit our website: 